\title{
Urolithiasis in a child with acute lymphoblastic leukemia - a case report and review of literature
}

\author{
Sangeetha Geminiganesan ${ }^{1}$, Niranjana Varadaraju², Latha M Sneha ${ }^{3}$, Julius Scott ${ }^{4}$ \\ ${ }^{1}$ Dr.Sangeetha Geminiganesan, Fellowship in Pediatric Nephrology, Assistant Professor, Department of Pediatrics, \\ Sri Ramachandra University, ${ }^{2}$ Dr. Niranjana Varadaraju, Post Graduate III year, MD Pediatrics. Department of \\ Pediatrics, Sri Ramachandra University, ${ }^{3}$ Dr. Latha M Sneha, MD Pediatrics, Assistant Professor, Division of \\ Pediatric Hemato Oncology, Department of Pediatrics, Sri Ramachandra University, ${ }^{4}$ Dr. Julius Scott, Professor of \\ Pediatrics, Head/Division of Pediatric Hemato Oncology. Department of Pediatrics, Sri Ramachandra University, \\ Chennai, Tamilnadu, India.
}

Address for Correspondence: Dr. Latha M Sneha, Assistant Professor, Division of Pediatric Hemato Oncology, Sri Ramachandra University, E mail id: drmslatha@yahoo.com

\begin{abstract}
A two years old girlwith newly diagnosed acute lymphoblastic leukemia on induction chemotherapy developed abdominal colic and passage of powdery material in the urine. Ultrasound abdomen revealed stone in the right kidney. She was treated with adequate hydration and alkali therapy and improved symptomatically. She has completed intensive phase of chemotherapy and had no recurrence of symptoms. Repeat ultrasound after two months of therapy showed no stones. Though renal stone disease is rare in childhood leukemia, it should be ruled out, as abdominal colic is generally attributed to the common side effects of drugs used in chemotherapy.
\end{abstract}

Key words: Child, Acute leukemia, Urolithiasis

\section{Introduction}

The incidence of renal stone disease in children is showing increasing trend due to various factors. Underlying metabolic problems are the most common cause of stone disease in children. Children with malignancy may be at more risk of developing stone during the therapy because of exposure to glucocorticoids, ectopic parathyroid hormone production and reduced physical activity. Common manifestations of stone are abdominal colic, hematuria and passage of stones. Here we present a child with acute lymphoblastic leukemia who developed abdominal colic during induction phase, turned out to be renal stones and treated conservatively.

\section{Case History}

A previously healthy, developmentally normal two years old girl child presented with the history of fever and cough for ten days and refusal to walk due to leg pain for one week. Investigations revealed pancytopenia and bone marrow aspirate flow cytometry was confirmatory of Pre B Cell Acute Lymphoblastic Leukemia (ALL).

As per standard risk strategy, she was started on 3 drug regimen, with prednisolone $60 \mathrm{mg} / \mathrm{m}^{2} /$ day, vincristine and Lasparaginase. The child had a stormy course in first 2 weeks of induction therapy. She developed pneumothorax and pneumomediastinum and needed left sided inter costal drainage. She also underwent Video Assisted Thoracoscopic Surgery for the management of multiple loculated fluid collections. After spending three weeks in ICU, she was transferred to ward when shecomplainedof abdominal pain.It was attributed tovincristineinduced paralytic ileus and hence an $\mathrm{x}$ ray taken to rule out constipation was normal.

Manuscript received: $6^{\text {th }}$ December 2017

Reviewed: $16^{\text {th }}$ December 2017

Author Corrected: $25^{\text {th }}$ December 2017

Accepted for Publication: $31^{\text {st }}$ December 2017 
Original Research Article

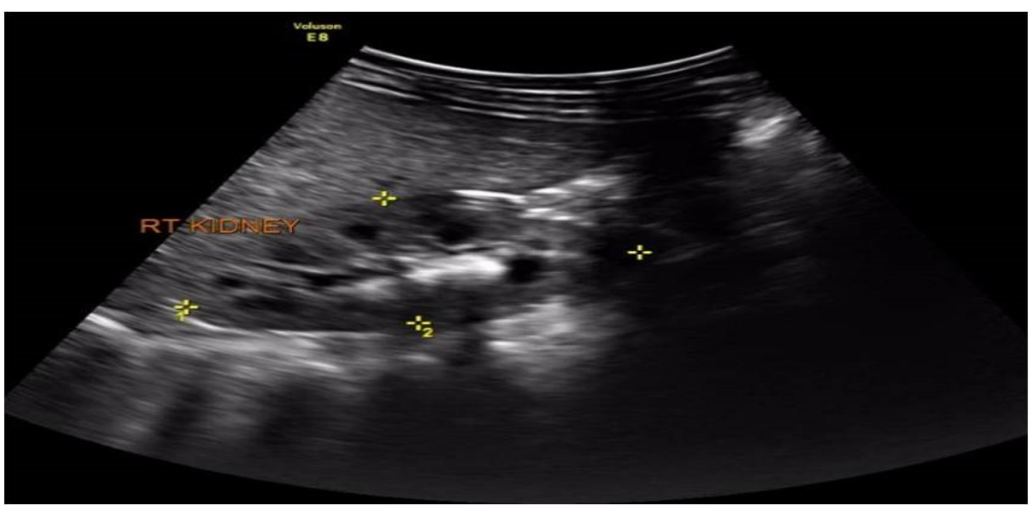

Figure 1:18 X $7 \mathrm{~mm}$ calculus at the right renal pelvis extending in to the calyces causing mild prominence of the pelvicalycealsystem.

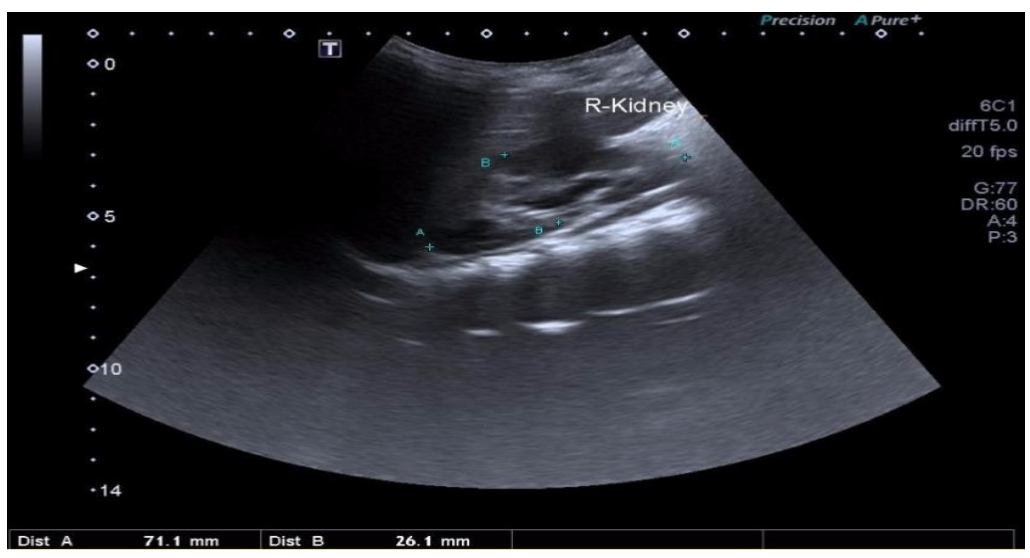

Figure-2: No calculus seen in the kidney

In view of persistence of abdominal pain and as mother noticed intermittent passage of powdery material in urine, USG abdomen was done which showed a $1.8 \times 0.7 \mathrm{~cm}$ stone in the right renal pelvis (fig 1 ). She did not have fever, hematuria, passage of stone, dysuria, urgency and increased frequency of urination. Urine routine, renal function test, total counts, serum calcium and uric acid levels done at this time were normal. Serum electrolytes were with in normal limits except for low bicarbonate levels $(18 \mathrm{mEq} / \mathrm{L})$. Spot urine calcium creatinine ratio was not contributory. As she had poor oral intake and intermittent vomiting, she was treated with intravenous fluids andalkali. As the renal stonesize was significant, parents were counseled for 24 hours crystalluria evaluation at a later date. Fortunately, she never developed recurrence of symptoms and alkali therapy was stopped after a couple of weeks. On follow up after threemonths, repeat ultrasound was normal with no evidence of renal stones (fig 2).

\section{Discussion}

An estimated prevalence of life time urolithiasis is 10 to $12 \%$ in men and 5 to $6 \%$ in women where as the incidence in children is only 1 to $2 \%$ [1]. It is one of the common causes of abdominal colic in children. Though the incidence of renal stone disease in children is not known accurately, it is showing an increased trend approximately by $6-10 \%$ [2].

Potential risk factors for urolithiasis in children are male sex, family history of renal stones, obesity, immobilization, glucocorticoid therapy, hyper- calciuria, hyperoxaluria, hypocitraturia, hyper uricosuria and urinary tract infection. It is proposed that reduced water intake, excess salt and fructose consumption, elevated environmental temperature leading to reduction in urine volume, allcauses increased urinary super saturation and stone formation. Structural malformation of the kidney, medications and toxins induced nephrolithiasis are also being reported in children. Howard et al had reported an incidence of $0.9 \%$ of urolithiasis among pediatric patients with ALL where as Mariane et al 
had reported an incidence of $3.4 \%$ among his study population of 12 children $[3,4]$. Pre disposing factors for renal stone in a child with malignancy are tumor lysis syndrome, long term steroids, prolonged immobilization, urinary alkalization and oral allopurinol during the initial periods of induction phase.

Tumor lysis syndrome releases large amounts of potassium, phosphate, and nucleic acids into the systemic circulation whose catabolismleads to hyperuricemia. Uric acid, calcium and phosphorous precipitate in the renal tubules and form stones.

The incidence of urolithiasis per 10000 person years among ALL children as quoted by Howard et al was 83 during induction therapy, 41 during continuation therapy with pulses of glucocorticoid and 9.3during continuation therapy without glucocorticoids and 1.8 after completion of all chemotherapy [3].

The most common symptoms of urolithiasis in pediatric population are heamaturia (33-90\%), abdominal or flank pain ( $94 \%$ in adolescents and $56 \%$ in 0-5 years of age) and urinary tract infection which is more common in preschool aged children [5].

Howard et al study showed hematuria in $88 \%$, abdominal or flank pain in $94 \%$, visualization of stone in $63 \%$ and dysuria in $47 \%$. He also reported thatan identifiable risk factor was found in almost $80 \%$ of his patients, where in $70 \%$ had received glucocorticoids within 90 days of diagnosis of urolithiasis and 35\% had been immobilized (defined as bed rest for 7 day or more) and $35 \%$ had a family history urolithiasis in a first degree relative.

In our patient, the predisposing factors were immobilization for more than a month, family history of urolithiasis (mother had asymptomtic renal stone), poor hydration status and glucocorticoid therapy. Glucocorticoid therapy over a long period may decrease the absorption of calcium from the gastrointestinal tract and cause hypercalciuria.

Evaluation for stone forming substances in the blood and urine are essential in children with nephrolithiasis as it may show positivity in 40 to $95 \%$ of them. Twenty-four hours urine for crystalluria evaluation is mandatory in children presenting with stone to look for hypercalciuria, hyperuricosuria and hypercalciuria. Generally children with nephro-

\section{Original Research Article}

lithiasis are encouraged to drink plenty of water which is the foremost important step in the prevention of stone formation. They should take minimum water intake of 1.5 to 2 litres $/ \mathrm{m}^{2} /$ day.

To prevent the super saturation of substances in the urine, output should be adequate greater than $750 \mathrm{ml} /$ day in infants, 1 litre/day in children less than 5 years, 2litre/day in older children and adolescents and greater than 3litre/day in late adolescent age group. Diet modification is by avoiding excessive consumption of fructose and glucose containing drinks, restriction of sodium intake and foods rich in oxalate like chocolates, spinach, sweet potatoes and nuts. Pharmacotherapy include alkali therapy with citrate, thiazide in hypercalciuria and $\mathrm{D}$ penicillamine in cystinuria. In children with significant larger size stones and obstructive signs, surgical intervention may be needed.

\section{Conclusion}

Although urolithiasis is rare in pediatric ALL, abdominal or flank pain, hematuria and urinary tract infections in such childrenshould promptto rule out renal stones as the etiology. As urolithiasis may delay the underlying disease managementdue to pain and hematuria, preventive measures and early diagnosis of urolithiasis are mandatory.

\section{What this study add to existing knowledge?}

Always keep renal stones as a differential diagnosis for children with ALL presenting with abdominal colic. Preventive measures in the form of proper hydration is essential. Timely imaging may be helpful to diagnose and treat the conditionearly.

Funding: Nil, Conflict of interest: None initiated, Perission from IRB: Yes

\section{References}

1. Romero V, Akpinar H, Assimos DG. Kidney stones: a global picture of prevalence, incidence, and associated risk factors. Rev Urol. 2010;12(2-3): e 8696.

2. Tasian GE, Copelovitch L. Evaluation and medical management of kidney stones in children. J Urol. 2014; 192 (5): 1329-36.doi: 10.1016/j.juro. 2014. 04.108 .

3. Howard SC, Kaplan SD, Razzouk BI, Rivera GK, Sandlund JT, Ribeiro RC et al. Urolithiasis in 


\section{Original Research Article}

pediatric patients with acute lymphoblastic leukemia. Leukemia 2003; 17(3): 541-46. DOI:10. 1038/sj. leu.2402852.

4. Camargo MGM, Duarte RJ, Cristofani LM, Filho VO and Srougi M. Urolithiasis in Childrn with Acute
Lymphoblastic Leukemia. Journal of Analytical Oncology. 2013; 2: e 160-64.

5. Gillespie RS,Stapleton FB. Nephrolithiasis in Children. Pediatrics in Review. 2004:25(4); 131-39.

\section{How to cite this article?}

Sangeetha Geminiganesan, Niranjana Varadaraju, Latha M Sneha, Julius Scott. Urolithiasis in a child with acute lymphoblastic leukemia - a case report and review of literature. Int J Pediatr Res. 2017;4(12):754-757.doi:10. 17511/ijpr.2017.i12.09. 